\title{
In ornamento totius palatii? Selektive Wahrnehmung der königlichen Entourage in frühmittelalterlichen Quellen
}

„Ich habe Dich bei Deinem Namen gerufen“ (Jes 43,1). Nicht nur in der Bibel ist die namentliche Nennung einer Person von großer Bedeutung. Auch in der mittelalterlichen Gesellschaft spielt der namentliche Ausruf oder die Niederschrift des Namens von Menschen eine wichtige Rolle. Dies ist zum Beispiel der Fall in der liturgischen Praxis der Erinnerung an die Verstorbenen und Lebenden, die sowohl in Diptychen ${ }^{1}$ als auch in Büchern ${ }^{2}$ festgehalten wurde, und woraus Historiker aufschlussreiche Informationen über den Zusammenhalt der mittelalterlichen Gesellschaft gewinnen können. ${ }^{3}$ Ebenfalls in anderen Bereichen ist die Nennung der Wirkenden von großer Relevanz - beispielsweise in Urkunden: Dort geht es nicht nur um den namentlichen Hinweis auf Personen, die am Beurkundungsvorgang beteiligt wurden, um Rechtssicherheit zu gewährleisten, ${ }^{4}$ sondern auch um das Schweigen über bestimmte Akteure, denn der Verzicht auf eine Nennung ist in politischer Hinsicht von großer Bedeutung. ${ }^{5}$

Der Ansatz, den ich hier verfolgen möchte, betrifft die narrativen Quellen aus der Karolingerzeit: Zum einen geht es darum zu verstehen, aus welchem Grund bestimmte Leute in diesem oder jenem Zusammenhang genannt werden und zum anderen darum nachzuvollziehen, warum die zu erwartende Nennung einer Person umgangen wird.

1 Siehe beispielsweise Rolf Bergmann, Die Trierer Namenliste des Diptychons Barberini im Musée du Louvre, in: Namenforschung. Festschrift für Adolf Bach zum 75. Geburtstag, hrsg. von Rudolf Schützeichel und Matthias Zender, Heidelberg 1965, 38-48.

2 Einen hervorragenden Überblick zum Forschungsstand bietet folgender Ausstellungskatalog: Peter Erhart und Jakob Kuratli (Hrsg.), Bücher des Lebens - lebendige Bücher, St. Gallen 2010.

3 Es sei beispielsweise auf die neue Deutung der Namenliste im Prager Sakramentar hingewiesen siehe Carl I. Hammer, The Social Landscape of the Prague Sacramentary: The Prosopography of an Eighth-Century Mass-Book, in: Traditio 54 (1999), 41-80; Stuart Airlie, Earthly and Heavenly Networks in a World in Flux: Carolingian Family Identities and the Prague Sacramentary, in: Max Diesenberger, Rob Meens und Els Rose (Hrsg.), The Prague Sacramentary: Culture, Religion, and Politics in Late Eighth-Century Bavaria, Turnhout 2016 (Cultural Encounters in Late Antiquity and the Middle Ages, 21), $203-224$.

4 Philippe Depreux, Bitte und Fürbitte am karolingischen Hof. Zugleich ein Beitrag zur politischen Bedeutung der Ambasciatoren- und Impetratorenvermerke (Mitte 8. bis Mitte 9. Jahrhundert), in: Archiv für Diplomatik, Schriftgeschichte, Siegel- und Wappenkunde 58 (2012), 57-101; Ders., Les mentions hors teneur des diplômes de rois et empereurs aux temps carolingiens, entre notes à l'usage des notaires et instruments de communication politique, in: Olivier Canteaut (Hrsg.), Le discret langage du pouvoir. Les mentions de chancellerie du Moyen Âge au XVIIe siècle, Paris 2019 (Etudes et rencontres de l'Ecole des chartes, 55), 41-75.

5 Geoffrey Koziol, The Politics of Memory and Identity in Carolingian Royal Diplomas. The West Frankish Kingdom (840 - 987), Turnhout 2012 (Utrecht Studies in Medieval Literacy 19).

Ә OpenAccess. (c) 2021 Philippe Depreux, published by De Gruyter. (cc) BY-NC-ND This work is licensed under the Creative Commons Attribution-NonCommercial-NoDerivatives 4.0 International License. 
Es ist zu bezweifeln, dass eine allgemein gültige Strategie erkannt werden kann; ich möchte vielmehr Beispiele analysieren, die die Vielfalt der Möglichkeiten aufzeigen, wie die Leute in der Karolingerzeit die Erinnerung an die Zugehörigkeit bestimmter Personen zur führenden politischen Elite hervorheben oder auch umgehen konnten.

\section{Dhuoda und die Erinnerungspraxis der Wilhelmiden}

Dass je nach Kontext unterschiedlicher Personen gedacht wird, liegt auf der Hand. Dies ist umso leichter nachvollzuvollziehen, wenn man - wie Dhuoda im 9. Jahrhundert - zwischen öffentlichen und privaten Angelegenheiten unterscheidet. Hier geht es tatsächlich um eine Auswahl. Dhuodas Liber manualis ist eine wertvolle Quelle für die Erforschung der frühmittelalterlichen memorialen Praxis innerhalb adliger Familien: Die Aufforderung, für die Toten der Familie zu beten, ist Bestandteil der guten Ratschläge der Mutter an ihren Sohn. ${ }^{6}$ Es wurde schon betont, dass die Aufrechterhaltung der genealogischen Beziehungen in Bezug auf die Erbschaft eine wichtige Motivation für das Totengedenken ist. ${ }^{7}$ Bemerkenswertweise spielt das Amt eine Rolle bei der Wahrnehmung der Toten: Im Werk ist die Aufforderung zu beten in zwei unterschiedliche Teile getrennt dargestellt. Das ganze Buch VIII ist dem Gebet gewidmet. Wilhelm soll für alle beten, insbesondere (der Reihe nach) für die kirchlichen Würdenträger, die Bischöfe und Priester, für die Könige und Hoheiten, für seinen eigenen Herrn (d.h. für König Karl den Kahlen) und für seinen eigenen Vater (Bernhard) sowie für das ganze Volk Gottes; schließlich soll Wilhelm auch für die verstorbenen Eltern seines Vaters, für seinen eigenen Patenonkel (Theoderich) und für sich selbst beten. ${ }^{8}$ Getrennt von dieser Aufforderung stellt Dhuoda eine Liste der Verstorbenen auf, für die Wilhelm beten soll. Diese Liste ist im vorletzten Buch (X) des Liber manualis überliefert. Es ist besonders bedauernswert, dass das Original nicht erhalten ist, ${ }^{9}$ denn die Überlieferung dieser Liste ist problematisch. Zwar wird sie in Buch VIII angekündigt, aber sie ist möglicherweise nicht wirklich Bestandteil des Werkes. Zu Beginn des Abschnittes über die Gebetspflicht Wilhelms gegenüber seinen Großeltern väterlicherseits schreibt Dhuoda: „Bete für die Eltern deines Vaters, die ihm ihr Vermögen

\footnotetext{
6 Patrick J. Geary, Phantoms of Remembrance. Memory and Oblivion at the End of the First Millennium, Princeton 1994, 49.

7 Régine Le Jan, Dhuoda ou l'opportunité du discours féminin, in: Cristina La Rocca (Hrsg.), Agire da donna: Modelli e pratiche di rappresentazione (secoli VI-X), Turnhout 2007 (Haut Moyen Âge 3), 109128, hier 118.

8 Dhuoda, Manuel pour mon fils, hrsg. von Pierre Riché, Bernard de Vregille und Claude Mondésert, Paris 1975 (Sources chrétiennes 225), 306-325; Dhuoda, Liber manualis. Ein Wegweiser aus karolingischer Zeit für ein christliches Leben, übersetzt von Wolfgang Fels, Stuttgart 2008 (Bibliothek der mittellateinischen Literatur 5), 138-148.

9 Zur handschriftlichen Überlieferung siehe Dhuoda, Manuel pour mon fils (Anm. 8), 45-50. Die älteste Handschrift (Nîmes, Bibliothèque du Carré d'Art, ms. 393) datiert vom späten 10. / 11. Jahrhundert.
} 
in gesetzlicher Erbfolge hinterließen. Wer sie gewesen sind oder wie ihre Namen lauteten, wirst Du in den Abschnitten am Ende dieses kleinen Buches verzeichnet finden“ (nomina eorum in capitulis huius libelli in fine inuenies conscripta). ${ }^{10}$ Die Liste aber, obwohl sie als $\S 5$ von Buch X überliefert ist, steht direkt nach dem Schlusseintrag: Finit hic liber manualis. Amen. Deo gratias. ${ }^{11}$ Auf Buch X folgt ein zusätzliches Buch (XI) über das Gebet der Psalmen, das mit einer ausführlichen Datierung (auf das Jahr 841) endet. Über den ursprünglichen Aufbau des Werkes wissen wir nichts, aber eines steht fest: Dhuoda unterscheidet zwischen dem Gebet aufgrund einer Pflicht in rechtlicher Hinsicht und dem Gebet für die Verstorbenen der Familie: „Hier erfahre kurz die Namen einiger Angehöriger, die ich weiter oben bei der Erwähnung anderer übergangen habe!“12 Wilhelms Patenonkel, Theoderich, wird an beiden Stellen namentlich erwähnt; in Buch VIII betont Dhuoda die Tatsache, dass Wilhelm dessen Erbe ist. ${ }^{13}$

\section{Einhards Blackout}

Im oben angeführten Beispiel geht es ausdrücklich um Erinnerung. Das Vergessen ist aber auch üblich - und ein absichtliches Vergessen scheint nicht selten zu sein. ${ }^{14}$ Ein Beispiel sehr geschickter damnatio memoriae liefert Einhard im 18. Kapitel seiner Vita Karoli:

Als [Karl] nach seines Vaters Tode mit seinem Bruder das Reich geteilt hatte, ertrug er dessen Feindschaft und Hass mit solcher Geduld, dass es allen bewundernswert erschien, wie er sich von ihm nicht einmal zum Zorn reizen ließ. Die Tochter des Langobardenkönigs Desiderius, die er dann auf seiner Mutter Geheiß geheiratet hatte, verstieß er wieder, man weiß nicht aus welcher Ursache, nach einem Jahre, und vermählte sich mit der Hildigard, einer Frau von hohem Adel aus dem Volk der Schwaben; diese gebar ihm drei Söhne [...].

Diese werden so wie die Töchter namentlich genannt. Auch die folgenden Frauen sowie die Beischläferinnen werden namentlich genannt - nur die Tochter des Langobardenkönigs nicht. ${ }^{15}$ Es steht außer Frage, dass Einhard den Namen kannte - nur aus politisch-diplomatischen Gründen nennt er ihn nicht. Ebenfalls Paschasius

10 Übersetzung: Dhuoda, Liber manualis (Anm. 8), 145; Zitat: Dhuoda, Manuel pour mon fils VIII, 14 (Anm. 8), 320.

11 Dhuoda, Manuel pour mon fils, X, 4 (Anm. 8), 352.

12 Dhuoda, Liber manualis X, 5 (Anm. 8), 163.

13 Dhuoda, Manuel pour mon fils (Anm. 8), 320 - 322; Übersetzung: Dhuoda, Liber manualis VIII, 15 (Anm. 8), 146-147.

14 Dazu siehe Johannes Fried, Der Schleier der Erinnerung: Grundzüge einer historischen Memorik, München 2004.

15 Einhard, Vita Karoli, Kap. 18, in: Reinhold Rau (Hrsg.), Quellen zur karolingischen Reichsgeschichte, Bd. 1, Darmstadt 1987 (Ausgewählte Quellen zur deutschen Geschichte des Mittelalters. Freiherr vom Stein-Gedächtnisausgabe 5), 188-189. 
Radbertus in seiner Vita Adalhardi ist vergesslich: Unde factum est, cum idem imperator Carolus desideratam Desiderii regis Italorum filiam repudiaret. ${ }^{16}$ „Es begab sich also, dass Karl der Große sich von der erwünschten Tochter des Desiderius trennte.“ Paschasius macht zwar ein Wortspiel mit dem Namen des Königs, benennt aber die Tochter nicht und es wäre falsch, davon auszugehen, dass sie Desiderata heißen konnte. ${ }^{17}$ Ihr Name wurde vergessen, weil Karls Ehe mit ihr politisch nicht mehr tragbar war.

\section{Erwähnen, ohne es zu tun}

Gelehrte am Hof und in den wichtigsten Bildungsstätten des karolingischen Reichs wussten nicht nur, ob, wie und wann sie - offen oder verdeckt mittels Pseudonymen ${ }^{18}$ - auf bestimmte Personen hinweisen sollten. Sie konnten auch geschickt über Andeutungen und literarische Tricks verschlüsselte Nachrichten verbreiten. Eine Gemeinsamkeit der Visio Wettini (um 824) und der Vision einer armen Frau aus Laon, die möglicherweise von Heito veranlasst wurde, ist es, dass dort Mitglieder der königlichen Entourage vorkommen. ${ }^{19}$ In der Vision der armen Frau werden einerseits Picho (Beggo), der Schwiegersohn Ludwigs des Frommen, und Ludwigs Frau, Irmgard, gequält, andererseits ist der Name Ludwigs - als Inschrift auf der Mauer der himmlischen Stadt - sehr schwer zu lesen, während der Name Bernhards von Italien in Goldbuchstaben erscheint. Es handelt sich um eine scharfe Kritik an Ludwig dem Frommen und seiner Politik in den ersten Jahren seiner Regierung. Das Vorkommen des im Jahr 816 verstorbenen Beggo, der vielleicht der engste Mitarbeiter des Königs in Aquitanien war, ${ }^{20}$ kann als Stellungnahme gegen Ludwig verstanden werden. Die

16 Vita Adalhardi 7, hrsg. von Georg Heinrich Pertz, in: MGH SS 2, Hannover 1829, 525. Zur Bedeutung der Auflösung des Bündnisses mit Desiderius und der Zurückweisung seiner Tochter nach Italien für die Laufbahn Adalhards siehe Verena Postel, „Communiter inito consilio“: Herrschaft als Beratung, in: Politische Reflexion in der Welt des späten Mittelalters. Political Thought in the Age of Scholasticism. Essays in Honour of Jürgen Miethke, hrsg. von Martin Kaufhold, Leiden 2004 (Studies in Medieval and Reformation Traditions 103), 1-25, hier 9.

17 Siegmund Hellmann, Desiderata, in: Neues Archiv der Gesellschaft für ältere deutsche Geschichtskunde 34 (1909), 208-209.

$18 \mathrm{Zu}$ dieser Praxis siehe u. a. Mary Garrison, The Social World of Alcuin: Nicknames at York and at the Carolingian Court, in: Luuk A. J. R. Houwen und Alasdair MacDonald (Hrsg.), Alcuin of York. Scholar at the Carolingian Court. Proceedings of the Third Germania Latina Conference Held at the University of Groningen, May 1995, Groningen 1998 (Germania latina 3 / Mediaevalia Groningana 22), 59-79.

19 Heito, Visio Wettini, hrsg. von Ernst Dümmler, in: MGH Poetae 2, Berlin 1884, 267-275; Walahfrid Strabo, Visio Wettini, ebd., 301-333; Walahfrid Strabo, Visio Wettini. Die Vision Wettis, übersetzt von Hermann Knittel, Sigmaringen 1986; Hubert Houben, Visio cuiusdam pauperculae mulieris. Überlieferung und Herkunft eines frühmittelalterlichen Visionstextes (mit Neuedition), in: Zeitschrift für die Geschichte des Oberrheins 124 (1976), 31-42.

20 Philippe Depreux, Prosopographie de l'entourage de Louis le Pieux (781-840), Sigmaringen 1997 (Instrumenta 1), $120-122$. 
Kritik von Mitgliedern der königlichen Entourage in der Visio Wettini ist etwas versteckter. Dank der Dichtung von Walahfrid Strabo sind wir über die Identität einiger Protagonisten informiert, denn ihr Name erscheint in Form von Akrostichen. Nur so ist gesichert, dass der König Italiens, der für seine Sünden büßen muss, eigentlich Kaiser Karl der Große (CAROLUS IMPERATOR) ist. ${ }^{21}$ Dort werden auch weltliche Großen genannt: ODALRIH und RUADRIH. ${ }^{22}$ Beide werden im Jenseits bestraft; ihre Mitbewohner werden sogar durch ihren Gestank doppelt bestraft, obwohl sie dort ständig im warmen Wasser baden. Graf Udalrich war ein Angehöriger einer wichtigen Familie, denn so hieß der Bruder Hildegards, der Gemahlin Karls des Großen und Mutter Ludwigs des Frommen. Zwischen 778 und 817 sind mehrere gleichnamige Grafen im Breisgau, Thurgau, Argengau und andernorts nördlich des Bodensees nachgewiesen, sodass es nicht möglich ist, sie genau zu unterscheiden. ${ }^{23}$ Vermutlich war Udalrich um 824/825 in St. Gallen nicht sehr gut angesehen. Bei dem Kumpan Udalrichs handelt es sich vielleicht um den Grafen in Rätien namens Roderich, der zwischen 823 und 829 von Bischof Viktor III. von Chur vor Kaiser Ludwig dem Frommen des Raubs von Kirchengut beschuldigt wurde. ${ }^{24}$ Es wird vermutet, dass dieser Roderich der Welfenfamilie, d.h. der Familie der Kaiserin Judith angehörte. ${ }^{25}$ Falls dies zutrifft, könnten zwei mit der Familie des Kaisers eng verwandte Grafen in St. Gallen um die Mitte der 820er Jahre in der Kritik der Mönche gestanden haben. ${ }^{26}$ Sie werden wahrscheinlich für alle Mönche erkennbar gewesen sein, nur muss man Walahfrids Schrift lesen (und nicht nur hören), um sie zu identifizieren.

21 Heito, Visio Wettini 11, 271; Walahfrid Strabo, Visio Wettini V. 446-461.

22 Heito, Visio Wettini 10, 270-271; Walahfrid Strabo, Visio Wettini V. 414-427, 317-318.

23 Michael Borgolte, Die Grafen Alemanniens in merowingischer und karolingischer Zeit: Eine Prosopographie, Sigmaringen 1986 (Archäologie und Geschichte 2), 248-254.

24 Michael Borgolte, Geschichte der Grafschaften Alemanniens in fränkischer Zeit, Sigmaringen 1984 (Vorträge und Forschungen 31), 219-229; Reinhold Kaiser, Churrätien im frühen Mittelalter, Basel 2008, 59-62.

25 Karl Schmid, Von Hunfrid zu Burkard: Bemerkungen zur rätischen Geschichte aus der Sicht von Gedenkbucheinträgen, in: Geschichte und Kultur Churrätiens. Festschrift für Pater Iso Müller OSB zu seinem 85. Geburtstag, hrsg. von Ursus Brunold und Lothar Deplazes, Disentis 1986, 181-209, hier 195 197.

26 Zum sozialen Umfeld dieser Nennungen siehe Philippe Depreux, Kaiserliche Amtsträger und Entourage Ludwigs des Frommen in und aus Alemannien und dem Elsass, in: Jürgen Dendorfer, Heinrich Maulhardt, R. Johanna Regnath und Thomas Zotz (Hrsg.), 817 - Die urkundliche Ersterwähnung von Villingen und Schwenningen. Alemannien und das Reich in der Zeit Kaiser Ludwigs des Frommen, Ostfildern 2016 (Veröffentlichung des Alemannischen Instituts Freiburg i. Br. 83 / Veröffentlichungen des Stadtarchivs und der Städtischen Museen Villingen-Schwenningen 39), 197-206. 


\title{
Achten auf die Etiquette: Der Reihe nach!
}

Das Epos De Karolo rege et Leone papa, das eine Art Bild „sur le vif“ der Familie Karls des Großen im Jahr 799 darstellt, beschreibt das Erscheinen Karls, seiner Frau und ihrer Kinder bei der Jagd:27

\begin{abstract}
Endlich tritt er hervor, umdrängt vom dichten Gefolge, Europas ehrwürdiger Leuchtturm schreitet ins Freie (V. 168f.). Nun schreitet aus ihrem stolzen Gemach die Königin, die lange verweilt, umdrängt von großem Gefolge, Liutgard mit Namen, Karls schöne Gemahlin (V. 182-184). Vom starken Gefolge geleitet, in Haltung und Gestalt dem Vater ähnlich, tritt endlich Karl heraus, der seines Vaters Namen trägt; er reitet wie gewöhnlich sein feuriges Ross (V. 196-199). Ihm folgt Pippin, benannt nach dem Großvater (V. 200). Darauf folgt die Reihe der herrlichen Töchter: Rhodrud, den andern voran, reitet stattlich einher auf schnellem Rosse (V. 212-214). In der Schar der Jungfrauen dahinter erscheint als nächste Berta, von vielen Mädchen geleitet (V. 219f.). Gisela folgt hinter diesen in schimmernder Weiße, vom Chor der Jungfrauen geleitet zieht einher die herrliche Prinzessin (V. 229f.). Rhodhaid erscheint alsdann im Glanz reichen Goldschmucks; freudig schreitet sie raschen Schrittes ihrem Gefolge voran (V. 243f.). Inzwischen tritt strahlenden Angesichts Theodrada heraus (V. 251). Das Ende des Zuges nimmt Hiltrud ein, ihr ist auch die letzte Gruppe des Hofes zugeteilt. Die Jungfrau selbst hält strahlend sich in ihrer Mitte. Sie hemmt nach kurzem Wege schon den raschen Schritt unweit vom Ufer des Flusses (V. 263-267).
\end{abstract}

Es wird ständig betont, dass die Mitglieder der königlichen Familie von einer Menge begleitet sind, sie schnell und fröhlich reiten, aber trotzdem geht es hier um eine wohl geregelte Ordnung, wie aus einem der letzten Verse dieser Beschreibung hervorgeht. Als er Hiltrud nennt, schreibt der Dichter: Agminis extremam partem sibi vindicat Hiltrud, / Illi sorte datur dehinc ultimus ordo senatus (V. 263f.). Hiltrud hat nur Anspruch auf die letzte Gruppe, weil das Schicksal es so bestimmt hat - denn: Hiltrud ist die letztgeborene der damals lebenden Töchter Karls des Großen. Bis auf die Tatsache, dass die beiden Söhne als erste genannt werden, entspricht die Reihenfolge genau dem Alter der Kinder. ${ }^{28}$ Es ist nicht verwunderlich, dass Ludwig der Fromme fehlt: Diese Jagdszene spielt sich im April 799 ab, denn währenddessen soll Karl der Große im Traum das Attentat gegen Leo III. gesehen haben. Wir wissen, dass Ludwig sich damals in Aquitanien aufhielt. ${ }^{29}$ Die Beschreibung des Hofes im Gedicht De Karolo rege et Leone papa steht nicht alleine da. Als weiteres Beispiel eines dem Alter ent-

27 Wilhelm Hentze (Hrsg.), De Karolo rege et Leone papa. Der Bericht über die Zusammenkunft Karls des Großen mit Papst Leo III. in Paderborn 799 in einem Epos für Karl den Kaiser, Paderborn 1999 (Studien und Quellen zur westfälischen Geschichte 36). Die Versangaben beziehen sich auf den lateinischen Text. Die Übersetzung von Franz Brunhölzl wurde dem Beiheft dieser Publikation entnommen.

28 Karl Ferdinand Werner, Die Nachkommen Karls des Großen bis um das Jahr 1000 (1.-8. Generation), in: Karl der Große. Lebenswerk und Nachleben, Bd. 4, hrsg. von Wolfgang Braunfels und Percy Ernst Schramm, Düsseldorf 1967, 403-482, hier 443.

29 Astronomus, Das Leben Kaiser Ludwigs, Vita Hludowici imperatoris 9-10, hrsg. von Ernst Tremp, in: MGH SS rer. Germ. 64, Hannover 1995, 308-309. 
sprechenden Auftritts von Königssöhnen kann man u.a. die Prognose über die Nachfolge Karls des Großen anführen, wie sie bei Ermoldus Nigellus vorkommt. Paulinus von Aquileia ist zu Besuch am Hof und beobachtet, wie die Söhne Karls des Großen in der ihrem Alter entsprechenden Reihenfolge die Kirche betreten: Zunächst kommt Karl, dann Pippin und zum Schluss Ludwig, der im Gegensatz zu seinen arroganten älteren Brüdern sich vor Gott demütigt. So habe der Patriarch von Aquileia erkannt, dass der jüngste Sohn Karls des Großen dazu geeignet sei, seinem Vater nachzufolgen. ${ }^{30}$

Zwar kann es vorkommen, dass in einer Auflistung aufgrund des Versmaßes die Reihenfolge dem Alter nicht entspricht, wie beispielsweise im Gedicht, das Theodulf von Orléans König Karl gewidmet hat, wo die Töchter Fastradas, Theodrada und Hiltrud, nach der älteren Tochter einer Konkubine in umgekehrter Reihenfolge genannt werden. ${ }^{31}$ Wenn dies nicht der Fall ist, muss man nach einer anderen, diesmal historischen Lösung suchen. Dies gilt zum Beispiel für die Beschreibung der Söhne Ludwigs des Frommen im Gedicht De imagine Tetrici des Walahfrid Strabo. ${ }^{32}$ Zunächst kommt Lothar (alias Josua), gefolgt von Ludwig dem Deutschen, der als Jonathan angeredet wird. Als dritter Edelstein (tertia gemma) wird Pippin erwähnt. Er war zur Abfassungszeit des Gedichtes nicht in Aachen, ${ }^{33}$ aber die Erwähnung an dritter Stelle ist merkwürdig. Sie ist es umso mehr als Walahfrid den Wunsch äußert, Pippin möge doch an Prestige nicht verlieren: Tertia gemma suos umquam non perdat honores. ${ }^{34}$ Dieses Gedicht wurde in der bewegten Zeit um das Ende der 20er Jahre verfasst, als Aquitanien der Ort war, wo die Opposition gegen die Politik Ludwigs des Frommen zum Ausdruck gebracht wurde bzw. wo einige Vorkommnisse instrumentalisiert

30 Ermold le Noir, Poème sur Louis le Pieux et épîtres au roi Pépin, hrsg. von Edmond Faral, Paris 1932, Les Classiques de l'histoire de France au Moyen Âge 14, 48-50 (I, V. 600 -647). Dazu siehe Philippe Depreux, Gestures and Comportment at the Carolingian Court: Between Practice and Perception, in: Michael J. Braddick (Hrsg.), The Politics of Gesture. Historical Perspectives, Oxford 2009 (Past \& Present Supplements, 4), 57-79, hier 74-76.

31 Theodulf, Carmen 25, V. 99, hrsg. von Ernst Dümmler, in: MGH Poetae 1, Berlin 1881, 486: Rothaidh poma, Hiltrudh Cererem, Tetdrada liaeum.

32 Walafrid Strabo, De imagine Tetrici, hrsg. von Ernst Dümmler, in: MGH Poetae 2, Berlin 1884, $375=$ Michael W. Herren, The „De imagine Tetrici“ of Walahfrid Strabo. Edition and translation, in: The journal of medieval Latin 1 (1991), 118-139, hier 126f. Zu diesem Gedicht siehe auch Ders., Walahfrid Strabo's De Imagine Tetrici: an interpretation, in: Richard North und Tette Hofstra (Hrsg.), Latin Culture and Medieval Germanic Europe, Groningen 1992 (Germania latina 1 / Mediaevalia Groningana 11), $25-41$.

33 Walafrid Strabo, De imagine Tetrici, V. $171 \mathrm{f}$. . (MGH) / V. $141 \mathrm{f}$. (Herren): Tertia gemma suos umquam non perdat honores, / De cuius meritis, quod non mihi visus inussit,/ Haurio florigena laetae dulcedine famae.

34 Friedrich von Bezold, Kaiserin Judith und ihr Dichter Walahfrid Strabo, in: Historische Zeitschrift 130 (1924), 377-444, hier 393, weist zwar auf diese Bezeichnung hin, deutet sie aber nicht. Zur möglichen Bedeutung dieser Auflistung siehe Depreux, Prosopographie (Anm. 20), 318. 
wurden. ${ }^{35}$ Diese Erwähnung Pippins von Aquitanien, die gegen die Etikette verstößt, könnte Gerüchte am Hof widerspiegeln.

\section{Zwischen Etikette und Wahrnehmung: Ingelheim 826}

Die Beschreibung der Hofgesellschaft, die bei Ermoldus Nigellus zu lesen ist, ist für unser Thema auch besonders interessant. Weder Graf Matfrid von Orléans noch Graf Hugo von Tours haben einen höfischen Titel getragen. Dies hinderte diese wichtigen Mitglieder der politischen Elite nicht daran, protokollarisch an prominenter Stelle aufzutreten, so in der Darstellung der Taufe Harald Klaks bei Ermoldus Nigellus. ${ }^{36}$ Der Dichter beschreibt den feierlichen Einzug des Kaisers in die Kirche der Pfalz Ingelheim: „Gänzlich von Golde bedeckt und funkelnd von edlen Gesteinen / Ging er des Weges gar froh und auf die Diener gestützt“. ${ }^{37}$ Ludwig stützt sich auf seine famuli, nämlich auf Hilduin, den damaligen Erzkaplan, und auf Helisachar, den ehemaligen Erzkanzler. Ihre Bezeichnung als famuli hebt ihre persönliche Bindung an den Herrscher hervor, denn dieser Ausdruck bezeichnet eigentlich Diener aus der familia. ${ }^{38}$ Der eine ist Mitglied der domus (er steht ihr sogar vor), der andere nicht (bzw. nicht mehr). Diese Beschreibung ist deswegen besonders interessant, weil sie zum Teil Leute erwähnt, die tatsächlich aufgrund ihres Amtes zu erwarten sind: Gerung als Türhüter, Fridugis als Erzkanzler; kurz vorher beschreibt Ermoldus die Gruppe der Geistlichen davon werden einige namentlich erwähnt. Es herrscht Eintracht und Ordnung - der Einzige, der diese Ordnung stören darf, ist der dreijährige Karl, der vor seinem Vater fröhlich herumhüpft. Diese Beschreibung des kaiserlichen Zuges ist aber keine Auflistung der Teilnehmer an der Reichsversammlung, die in Ingelheim im Juni 826 stattfand: Aus anderen Quellen wissen wir, dass Gesandte des Papstes (nämlich Bischof Leo von Civita Vecchia und der Nomenclator Theofilactus), aber auch sowohl Grafen aus der bretonischen Mark als auch der bayerische Markgraf Gerold und der

35 Zum politischen Kontext siehe Philippe Depreux, Der karolingische Hof als Institution und Personenverband, in: Le corti nell'alto medioevo, Bd.1, Spoleto 2015 (Settimane di studio della fondazione Centro italiano di studi sull'alto medioevo, 62), 137-164, hier 152-163.

36 Ermold le Noir, Poème sur Louis le Pieux (Anm. 30), 176. Dazu siehe Philippe Depreux, Le comte Matfrid d'Orléans sous le règne de Louis le Pieux, in: Bibliothèque de l'Ecole des Chartes, 152 (1994), 331-374. Ich bin mit der Deutung von Alain Dubreucq, Les peintures murales du palais carolingien d'Ingelheim et l'idéologie impériale carolingienne, in: Hortus artium medievalium 16 (2010), 27-38, nicht einverstanden.

37 Ermold le Noir, Poème sur Louis le Pieux V. 2292f., 176: Resplendens auro nimium gemmisque refulgens, / Innixus famulis laetus abibat iter. Übersetzung ins Deutsche: Theodor Gottfried Pfund und Wilhelm Wattenbach, Ermoldus Nigellus Lobgedicht auf Kaiser Ludwig und Elegien an König Pippin, Leipzig 1889 (Die Geschichtsschreiber der deutschen Vorzeit 2, 18), 86.

38 Isidori Hispalensis episcopi etymologiarum sive originum libri XX, hrsg. von Wallace Martin Lindsay, Bd. 1, Oxford 1911, 374 (IX, IV, 43): Famuli sunt ex propria servorum familia orti. 
friaulische Markgraf Baldricus anwesend waren. ${ }^{39}$ Es ist nicht vorstellbar, dass sie alle von der Feier ferngehalten wurden: Daraus ergibt sich also, dass Ermoldus nicht den Hof beschreiben, sondern eine politische Nachricht vermitteln will und dabei nur die in seinen Augen wichtigsten Protagonisten aus- und erwählt.

\section{Selektive Wahrnehmung}

Nachdem er die Ernennung Ludwigs des Frommen zum König von Aquitanien erwähnt hat, berichtet der Astronom, wie Karl der Große das politische Personal ersetzt hat:

Außerdem setzte er in ganz Aquitanien Grafen, Äbte und viele andere, die man gewöhnlich Vassi nennt, aus dem Volk der Franken ein, deren Klugheit und Stärke keiner mit schlauer List oder Gewalt gefahrlos hätte begegnen können; ihnen übertrug er die Sorge um das Reich, wie er es für nützlich hielt, die Sicherung der Grenzen und die Verwaltung der königlichen Hofgüter. In Bourges setzte er zuerst Humbert, bald darauf Sturbius als Grafen ein, in Poitiers Abbo, im Périgord Widbod, in der Auvergne Iterius, im Velay Bullus, in Toulouse Chorso, in Bordeaux Sigwin, im Albigeois Haimo und in Limoges Rotgar. ${ }^{40}$

Obwohl der Astronom behauptet, diese Maßnahme habe ganz Aquitanien betroffen, ist diese Auflistung sehr mangelhaft: Dort werden weder ein Bischof noch ein Abt, nur einige Grafen erwähnt. Wir können nicht beurteilen, ob viele Ortschaften, wie beispielsweise Cahors, Rodez, Saintes oder Angoulême nicht erwähnt werden, weil es dort keinen Anlass gab, die Amtsinhaber zu ersetzen, oder weil diese Franken waren. Dass Amtsinhaber anderer ethnischer Gruppen dem karolingischen Königshaus treu sein konnten, beweist der Vater Benedikts von Aniane, denn der Graf von Maguelonne war gotischer Abstammung. ${ }^{41}$ Leider fehlt in vielen Fällen jede schriftliche Überlieferung über diese Leute. Im letztgenannten Fall von Angoulême klagte schon der Verfasser der Geschichte der Bischöfe und Grafen von Angoumois im 12. Jh., dass seine Quellenlage sehr ungünstig sei. ${ }^{42}$

39 Siehe Johann Friedrich Böhmer, Die Regesten des Kaiserreichs unter den Karolingern 751-918, neubearbeitet von Engelbert Mühlbacher, nach Mühlbachers Tod vollendet von Johann Lechner, mit einem Vorwort, Konkordanztabellen und Ergänzungen von Carlrichard Brühl und Hans Heinrich Kaminsky, Nachdruck der 2. Aufl. (Innsbruck 1908), Hildesheim 1966, 325 (Nr. 829[770]b).

40 Astronomus, Das Leben Kaiser Ludwigs 3 (Anm. 29), 290 - 293.

41 Dazu siehe Depreux, Prosopographie (Anm. 20), $224 \mathrm{f}$.

42 Historia pontificum et comitum Engolismensium, hrsg. von Jacques Boussard, Paris 1957 (Bibliothèque elzévirienne. NS. Etudes et documents), 4: Civitas vero Engolismensium, negligentia scriptorum, ut credimus, memoriae non tradit facta subsequentium episcoporum a Mererio usque ad Launum, das heißt vom späten 6. Jh. bis zur Mitte des 9. Jh. Robert Favreau, Évêques d'Angoulême et Saintes avant 1200, in: Revue historique du Centre-Ouest 9 (2010), 7-142, hier 15, bestätigt diese ungünstige Quellenlage. 
Trotz der Kargheit der Quellenlage sind wir über andere Grafen in Aquitanien informiert. Eine interessante und einzigartige Quelle ist eine Urkunde Ludwigs des Frommen, mit der der König von Aquitanien am 3. August 794 in Jouac die Immunität der zum Kloster Saint-Hilaire in Poitiers zugehörigen Zelle von Nouaillé bestätigte. ${ }^{43}$ Diese im Original erhaltene Urkunde ist insofern einzigartig, als sie von Bischof Reginpert (von Limoges), der als Kaplan Ludwigs fungierte, und von 16 weiteren Zeugen durch ihr signum bekräftigt wurde. ${ }^{44}$ Einige sind als Mitglieder des Hofes nachweisbar, wie beispielsweise Adalbert (als Marschall) oder Erlaldus (als Seneschall). Möglicherweise sind einige dieser Subskribenten auch Grafen, wie beispielsweise der erste von ihnen, Magnarius, der vielleicht identisch mit dem von Karl dem Großen seinem Sohn Ludwig zugewiesenen Berater ist; ein gleichnamiger Graf ist in Narbonne nachweisbar. Immo ist wahrscheinlich Graf von Périgueux gewesen (der Astronom nennt nur Widbod). Es macht keinen Sinn, diese Liste bis ins letzte Detail hier durchzugehen: Es sollte lediglich gezeigt werden, dass andere Namen von Grafen zu Beginn der Regierungszeit Ludwigs des Frommen in Aquitanien bekannt sind.

Als weiteres Beispiel könnte man Ermolds Beschreibung der Belagerung Barcelonas in den Jahren 800 - 801 anführen: Der Dichter nennt Wilhelm, Heripreth, Liuthard, Bigo, Bero, Santio, Libulf, Hilthibreth und Isimbard. An andrer Stelle berichtet der Astronom über Liuthard: Im Jahr 803 wurde er zum Grafen von Fézensaz (in der Gascogne) gemacht. Als Sohn Graf Gerhards von Paris und Bruder Begos, des engsten Beraters und Freunds Ludwigs des Frommen während seiner Herrschaft in Aquitanien, gehörte Liuthard zweifelsohne zu einer dem Königshaus sehr getreuen Familie.

Es stellt sich also die Frage, warum der Astronom die Grafenliste im dritten Kapitel in dieser Form aufgestellt hat. Einige dort erwähnte Leute sind problematisch - und die Liste ist möglicherweise nicht in allen Punkten vertrauenswürdig. So beispielsweise im Fall von Bourges: Graf Humbert wurde wahrscheinlich nicht von Karl dem Großen eingesetzt; es ist nicht auszuschließen und es spricht sogar vieles dafür, dass er identisch mit dem Grafen von Bourges namens Unibert ist, ${ }^{45}$ der sich im Jahr 761 mit Waifar verbündete, wie in der Fortsetzung der Chronik des Fredegar zu lesen ist. ${ }^{46}$ Zur Bestrafung wurde Unibert samt reliqui Vascones (also doch kein Franke?) nach Francia versetzt, nachdem er einen Eid geleistet hatte. ${ }^{47}$ Einige Jahre später wird Unibert wieder als Graf von Bourges bezeugt; diesmal gilt er als Verbündeter Pippins gegen

43 Die Urkunden Ludwigs des Frommen, hrsg. von Theo Kölzer, in: MGH, Die Urkunden der Karolinger 2, Wiesbaden 2016, Bd. 1, 1-5 (Nr. 1).

$44 \mathrm{Zu}$ den Identifikationen verweise ich auf Depreux, Prosopographie (Anm. 20), passim.

45 Brigitte Kasten, Laikale Mittelgewalten: Beobachtungen zur Herrschaftspraxis der Karolinger, in: Franz-Reiner Erkens (Hrsg.), Karl der Große und das Erbe der Kulturen, Berlin 2001, 54-66, hier 61. 46 Fredegarii chronicorum liber quartus cum continuationibus. The Fourth Book of the Chronicle of Fredegar With Its Continuations, hrsg. von John Michael Wallace-Hadrill, London 1960, 110. (Continuationes 42).

47 Fredegarii chronicorum liber quartus cum continuationibus 43 (Anm. 46), 112. 
Waifar. ${ }^{48}$ Sein Nachfolger, Sturbius, auch vom Astronom erwähnt, ist ebenfalls keine unumstrittene Persönlichkeit: Adrevald von Fleury, der von seiner Ernennung im Zusammenhang mit dem Aufstand Pippins des Buckligen berichtet, bedauert seine unfreie Herkunft, allerdings interessiert ihn nur sein ebenso als Unfreier geborener Amtskollege Raho von Orléans, weil Fleury Opfer seiner listigen Gierigkeit gewesen sei. ${ }^{49}$ Auch andere vom Astronom genannte Grafen haben sich nicht als getreue Unterstützer der karolingischen Herrschaft in Aquitanien erwiesen: Chorso von Toulouse verband sich im Jahr 787 mit einem Basken, wie der Astronom zwei Kapitel später berichtet. ${ }^{50}$ Im folgenden Jahr wurde Chorso während einer Reichsversammlung in Worms abgesetzt und durch Wilhelm von Gellone, dessen Urgroßmutter eine Schwester Plectrudis‘, der Frau Pippins von Herstal, war, ersetzt. ${ }^{51}$ Der Astronom weiß also, was aus Chorso wurde. Des Weiteren weiß der Astronom über den Grafen von Bordeaux an anderer Stelle zu berichten: Im Jahr 816 rebellierten die Basken, weil ihr Graf, Sigwin, aufgrund seines „schlechten, ja fast unerträglichen Lebenswandels“ abgesetzt worden war. ${ }^{52}$ Was war also die Absicht des Astronomus? Nicht über alle von ihm aufgelistete Grafen kann man schlecht reden, aber über einige schon! Dass die Liste im dritten Kapitel nicht vollständig ist, ist eindeutig. Nach welchen Kriterien hat der Verfasser seine Wahl getroffen? Es ist schwer zu sagen. Diese Schrift ist eine Apologie für Kaiser Ludwig: Darf diese Liste als Mittel betrachtet werden, die Entscheidungen Karls des Großen im Schatten des Lobes zu kritisieren oder zu relativieren, weil auch er fehlbar war?

\section{Schlusswort: Hinkmar von Reims und der Glanz des Hofes}

Zum Schluss sei auf das Beispiel eingegangen, das dem Titel dieses Beitrags zugrunde liegt. In seinem 882 für König Karlmann verfassten Werk De ordine palatii erhebt Hinkmar den Anspruch, den Hof zu beschreiben, wie er in ornamento totius palatii in

\footnotetext{
48 Fredegarii chronicorum liber quartus cum continuationibus 51 (Anm. 20), 118.

49 Les miracles de Saint Benoît, hrsg. von Eugène de Certain, Paris 1858, 43f. (Miracula I, 18): ... quibusdam servorum suorum, fisci debito sublevatis, curam tradidit regni; atque in primis, Rahonem Aurelianensibus comitem praefecit, Biturigensibus Sturminium, Arvernis Bertmundum, aliisque, ut ei visum est, locis alios praeposuit. Sed Raho, ut ejus debitum exposcebat naturae, nimia insolescens astutia, cupiditate vero crudelior effectus, cum universa quae Aurelianensium fines continere videbantur sibi subjecta cerneret, coenobio duntaxat $S$. Benedicti excepto, quod ab abbate tunc regebatur Radulfo, consilium habuit, abbate interfecto, praefatum praeripere coenobium; quod maturato peregisset, nisi pietas indulgentissimi Conditoris obviam se tanto facinori opponere disposuisset. Dazu siehe Dominique Barthélemy, Chevaliers et miracles. La violence et le sacré dans la société féodale, Paris 2004, 129. 50 Astronomus, Das Leben Kaiser Ludwigs 5 (Anm. 29), 296.
}

51 Astronomus, Das Leben Kaiser Ludwigs 5 (Anm. 29), 298.

52 Astronomus, Das Leben Kaiser Ludwigs 26 (Anm. 29), 364. 
der Zeit Karls des Großen und Ludwigs des Frommen festgelegt wurde. ${ }^{53}$ Hinkmar erwähnt viele Ämter (nicht alle), nennt aber nur wenige Leute: nur die Erzkapläne, von Fulrad bis Drogo. Warum nur sie? Die Antwort scheint einfach zu sein: Es geht in dieser Schrift um Autorität. Hinkmar beruft sich auf die Autorität derer, die er möglicherweise gerne als seine Vorgänger betrachtet hätte, um seine eigene Autorität zu sichern bzw. sogar zu retten. ${ }^{54}$ Es ist möglicherweise ein Grund, warum er sich nicht auf die Zeit Karls des Kahlen beruft. Auch Hinkmars Gedächtnis ist selektiv - und dient seinem eigenen Ruhm.

53 Hinkmar von Reims, De ordine palatii, hrsg. von Thomas Gross und Rudolf Schieffer, in: MGH Fontes iuris 3, Hannover 1980, 56.

54 Dazu siehe Steffen Patzold, Konsens und Konkurrenz. Überlegungen zu einem aktuellen Forschungskonzept der Mediävistik, in: Frühmittelalterliche Studien 41 (2007), 75-103, hier 77-88. 